\title{
Author Index Vol. 1,1994
}

Agro,A. 350 Airaghi,L. 42,321 Aksentijevich, S. 66 Allolio,B. 14 Altemus,M. 242 Arbitrio,M. 135,141,174 Arimura, A. 127,335 Arkins, S. 33 Arit,W. 14 Assenmacher, I. 292 Audino,M.G. 361

Barbanel,G. 292 Belova,N. 86,116,195 Berczi,I. 201 Berkenbosch, F. 300 Bemat,A. 242 Biragyn,A. 33 BIalock,J.E. 50 Boissin.J. 292 Bongiorno, P.B. 242 Borda,E. 284 Brabant, G. 242 Brady, L.S. 59 Bredow,S. 100 I\&Ji,A.T. 357 Bristow,A.F. 292

Capetti,A. 42 Cardinali, D.P. 265 Carino, M. 315 Caroleo,M.C 135,141,174 Catania, A. 28,42,93,121,321 Ceriani,G. 28,121 Chisari,A.N. 315 Christian, C. 159 Chrousos, G.P. 14,274 Chung, H.-T. 377 Cizza,G. 231 Cousins, S.W. 188 Crofibrd, L.J. 59

Dantzer,R. 33 Davies,D. 308 Dees,W.L. 2,251 Demissie,S. 278 DeSarro,G. 361 Diaz, J. 28 Djergovid,D. 236

Esquifino,A.I. 265

Fang, J. 100 Fellows, M.Q. 66 Floyd, R. 100

Gaillard,R.C. 315 Gajewski,M. 259 Gerloni,V. 321 Ghanta,VX 74,278,370 Gilbert,|M. 159 Gimeno,M. 2,86,116,195 Givalois,L. 292 Goin,J.C. 284

Gold,P.W. 59,66,231,242,274 Gomez,,M. 231 Gottschall,P.E. 127 Grabczewska,E. 259 Gratteri,S. 361 Greibler,C. 231 Grossi, L. 321 Grossman, A. 23,329 Gushchin,G.V. 259

Hadid,R. 315 Haynes,L.W. 308 Heppner, C. 14 Hiney,J.K. 251 Hiramoto,N. 278 Hiramoto,R.N. 74,278,370 Hsueh, C.-m. 74,370 Huang, Q.-H. 335

Ixart,G. 292

Jakovleva,E.E. 259 Jankovid, B.D. 236 Jun,C.-D. 377

Kamat, A. 2,86 Kapas,L. 100 Karanth,S. 2,82 Kataeva,G. 350 Kataeva,G.V. 259 KeUey,K.W. 33 Kim,H.-M. 377 Kim,J.-M. 377 Komaki,G. 127 Komorowski, J. 149 Korneva,EA. 259 Krueger,J.M. 100

Laskowska-Bozek,H. 259 Licinio,J. 110,181,242 Upton, J.M. 28,42,93,121,321

Liu,Q. 33

Locatelli.L. 357

Lomater, C 321

Love,L.A. 59

Lumanglas, A.A. 343

Lynn,A.B. 59

Lyson,K. 2,82,86,121,153,251

McCann, S.M. 1,2,82,86,116,153,195,

251 Makara,G.B. 300 Malaval,F. 292 Manfredi,M.G. 42,321 Masek,K. 165 Maslinski,W. 259 Maurel,D. 292 Mbulamberi, D. 14 Mekaouche, M. 292 Melchiorri, D. 141 Michelson, D. 274 MiIazzo,F. 42 Miljevid, C. 236 Minshall,C. 33 MirteUa, A. 23 MisiewiczPoltorak, B. 59 Murpbree, S. 28

Navarra,P. 23

Nistic6,G. 135,141,174,361

Obdl,F.,Jr. 100 Omeljaniuk, R.J. 66

Page,S.W. 59 Panerai,A.E. 357 Paraet,P. 33 Pawlikowski, M. 149 Payan,D.G. 159 Perez Leiros, C. 284 Petrovicky, P. 165 Petzke,F. 14 Pozzoli, G. 23 Preziosi,P. 23 Procaccia,S. 321 Pulvirenti, L. 135,174

RaderJX. 59 Radulovic,J. 236,357 Raybourne, R.B. 59 Reincke,M॥ 14 Rettori,V. 2,86,116,195,251 Rim,G.-N. 377 Rogers, CF. 278,370 
Rotudfi.0. 361

RyzewskiJ. 259

Whitfield, H J., Jr. 66

Sacerdote,P. 357

Schotanus,K. 300

110,181,242

Seifert,J. 165

Siaud,P. 292

Siekmann,L. 14

Smith, C.C. 59,66,231

Smith, H. 308

Somogyvari-Vigh,A. 127,335

Spicer,BA. 308

Spinedi,E. 315

Stanisz, A.M. 217,350

Stepien,H. |M

Sterin-Borda, L. 284

Sternberg, E.M. 59,66,231
*'Streilein,J.W. 188

Suescun,M. 315

Takaki,A. 335

Takashima,A. 121

Tamarkin,L. 242

Tatsuno,I. 127

Taylor,A.W. 188

Thomas, F.S. 59

Tilders, F.J.H. 300

Toth,L.A. 100

Tsagarakis, S. 329

Tyring, S.K. 74

Vivirito,M.C. 42

Vollmer,D, 14
Wang,B.S. 343

Weigent,D.A. 50

Winkelmann, W. 14

Wong,M.-L.

Yatohgo,T. 127

Yoon, H.-J. 377

Yu.W.H. 195

Zanussi,C. 42

Zelazowska, E. 66

Zelazowski, E. 59

Zelazowski, P. 59

Zhang, R.-j. 343 
Author

Index 